\title{
The prevalence and morphometric features of mastoid emissary vein on multidetector computed tomography
}

\author{
G. Demirpolat, E. Bulbul, B. Yanik \\ Department of Radiology, Faculty of Medicine, Balıkesir University, Turkey
}

[Received: 29 October 2015; Accepted: 17 December 2016]

Background: The aim of the study was to evaluate the prevalence and morphometric features of mastoid emissary vein (MEV) on multidetector computed tomography (MDCT) scans, emphasize its clinical significance and review its surgical implications.

Materials and methods: Cranial and temporal bone MDCTs of 248 patients (496 sides) were analysed by 2 radiologists. Mastoid foramen (MF) was defined on the 3 dimensional volume rendered (3DVR) images. The MF and mastoid emissary canal (MEC) were investigated in axial thin slices and the diameters of the largest MF and MEC were measured. Mean diameters of MF and MEC were determined. The number of the mastoid foramina was noted. Differences in MF prevalence by sex and side were evaluated.

Results: The overall prevalence of MEC was $92.3 \%$. It was observed in $91.5 \%$ of women and $93.3 \%$ of men. MEC was present on the right side in $84.7 \%$ and on the left side in $82.3 \%$ of temporal bones. The mean diameter of MF was $1.92 \pm$ $\pm 1.02 \mathrm{~mm}$ on the right and $1.84 \pm 0.98 \mathrm{~mm}$ on the left. In both sides the number of the MF's changed from absent to triple. The mean diameter of MEC was $1.58 \pm 0.86 \mathrm{~mm}$ on the right and $1.48 \pm 0.79 \mathrm{~mm}$ on the left side. The mean diameter of MEC was significantly larger in men. No significant correlation was detected between age and the MEC diameter.

Conclusions: The preoperative detection of mastoid emissary veins is necessary. The radiologists should be familiar with their clinical significance and variant appearances and report them accurately. Knowledge of their morphology and surgical implications by the surgeons will make them aware to avoid unexpected and fatal complications while operating in the suboccipital and mastoid area. MDCT is a reliable diagnostic tool for imaging the MEC and MF. (Folia Morphol 2016; 75, 4: 448-453)

Key words: emissary vein, mastoid emissary canal, mastoid foramen, multidedector computed tomography

\section{INTRODUCTION}

Emissary veins traverse the skull by foramina and establish direct connections between dural venous sinuses and superficial veins of the scalp $[2,5,11,23]$.
Although these vessels lack valves and blood may flow in both directions, blood flow is generally from external to internal $[5,11,16]$. Along with the internal jugular veins, they participate in extracranial

Address for correspondence: Dr G. Demirpolat, Department of Radiology, Faculty of Medicine, Balıkesir University, Turkey, e-mail: gulendemirpolat@hotmail.com 


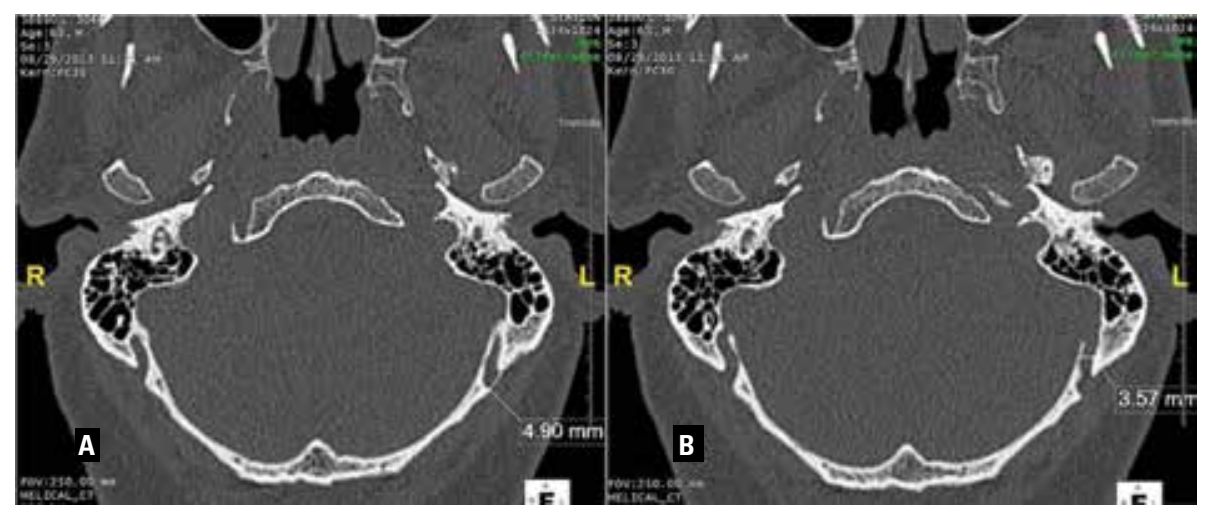

Figure 1. Non-enhanced axial computed tomography scan in a patient showing the diameter of the mastoid foramen (A) and the diameter of the canal of the mastoid emissary vein (B).

venous drainage of the neurocranium. When the normal routes of venous drainage are patent, the role of emissary veins is limited. They may be the primary outflow pathway in patients with intracranial hypertension, high-flow vascular malformations or hypoplasia/aplasia or occlusion of the internal jugular veins $[10,11,16,17,22]$. The mastoid emissary vein (MEV), posterior condylar vein, occipital emissary vein, and petrosquamosal sinus (PSS) are the major posterior fossa emissary veins of clinical importance.

The MEV runs between the sigmoid sinus and posterior auricular or occipital veins and provides venous drainage of the cerebral structures to suboccipital venous plexus and vertebral venous system [11, 17, 22]. It arises from the sigmoid sinus, courses in the mastoid emissary canal (MEC) and emerges from a foramen, the mastoid foramen (MF), which is located behind the mastoid process and may be situated around or in the occipitomastoid suture [10,16, 22].

Mastoid foramen, MEV, and MEC were examined in morphological and anatomical studies. Its functional and physiologic importance, and its implications in operative planning for the plastic and reconstructive surgeons have been investigated in a recently published study [11]. The radiological investigation of MEVs is limited and they are often overlooked on imaging studies. Multidetector computed tomography (MDCT) is a valuable tool for assessing the skull base and the skull base foramina. Very small emissary canals can be visualised with their exact course. Foramina and emissary canals can indicate the topography of the emissary veins and guide the radiologists in estimating their course for the further clinical implications. Here, we review the anatomy of the MEC and the MF based on high-resolution MDCT scans and emphasize their clinical relevance under the light of the literature.

\section{MATERIALS AND METHODS}

This retrospective study had institutional review board approval (IRB number 2014/73).

Images of non-contrast head and temporal bone thin-section MDCTs of 248 patients (129 women, 119 men; aged 5-89 years, mean age: $45 \pm 43$ years) were reviewed by 2 radiologists. All MDCTs had various clinical indications. Patients with posterior fossa, neck or temporal bone surgery, tumour and trauma were excluded from the study.

Head and temporal bone MDCTs were performed at 64-slice MDCT scanner (Toshiba Aquillon 64, Ottawara, Japan). Two-dimensional multiplanar reconstruction images and three-dimensional surface volume rendered images (3DVR) were reconstructed on TeraRecon workstation (AquariusNET Workstation 4.4.6; Toshiba Medical Systems). MDCT scan acquisition parameters for temporal bone computed tomography (CT) was $0.5 \mathrm{~mm}$ slice thickness, $120 \mathrm{kVp}, 200 \mathrm{~mA}$, and rotation time of $0.5 \mathrm{~s}$. Highresolution temporal bone MDCT scan was postprocessed with a bone algorithm. Head CT was conducted as $0.5 \mathrm{~mm}$ slice thickness, $120 \mathrm{kvP}, 280 \mathrm{~mA}$, and rotation time of $0.75 \mathrm{~s}$ and was reconstructed with bone algorithm. Axial slices were magnified and examined with coronal reformatted images when required.

The retrospective analysis of the MDCT scan slices was performed by 2 experienced radiologists. After review of the MF on 3DVR images, axial slices were investigated and if a MF did not connect with a canal, it was accepted as a pit not a foramen and was not included in the study. The diameters of the MEC and MF were measured (Fig. 1A, B). When more than one 
Table 1. The diameters of the mastoid emissary canal according to the side

\begin{tabular}{lcccc}
\hline Side & \multicolumn{4}{c}{ The diameter of the mastoid emissary canal } \\
\cline { 2 - 4 } & Absent & $<1 \mathbf{~ m m}$ & $\mathbf{1 - 3} \mathbf{~ m m}$ & $>\mathbf{3 ~ m m}$ \\
\hline Right $(\mathrm{n}=248)$ & $38(15.3 \%)$ & $48(19.4 \%)$ & $148(59.7 \%)$ & $14(5.6 \%)$ \\
Left $(\mathrm{n}=248)$ & $44(17.7 \%)$ & $55(22.2 \%)$ & $139(56.0 \%)$ & $10(4.0 \%)$ \\
Total $(\mathrm{n}=496)$ & $82(16.5 \%)$ & $103(20.7 \%)$ & $287(57.9 \%)$ & $24(4.8 \%)$ \\
\hline
\end{tabular}

Table 2. The four groups of mastoid foramina according to their diameters

\begin{tabular}{lcccc}
\hline Side & \multicolumn{4}{c}{ The diameters of the mastoid foramina } \\
\cline { 2 - 4 } & Absent & $<2 \mathbf{m m}$ & $2-4 \mathbf{~ m m}$ & $>4 \mathbf{~ m m}$ \\
\hline Right $(\mathrm{n}=248)$ & $38(15.3 \%)$ & $115(46.4 \%)$ & $88(35.5 \%)$ & $7(2.8 \%)$ \\
Left $(\mathrm{n}=248)$ & $44(17.7 \%)$ & $125(50.4 \%)$ & $74(29.8 \%)$ & $5(2.0 \%)$ \\
Total $(\mathrm{n}=496)$ & $82(16.5 \%)$ & $240(48.4 \%)$ & $162(32.7 \%)$ & $12(2.4 \%)$ \\
\hline
\end{tabular}

MF and canal were present the diameters of the largest ones were measured and they were referred as the diameter of the MEC and the diameter of the MF. The mean diameters of the MEC and MF were calculated. The numbers of MFs on each temporal bone were determined.

\section{Statistical analysis}

Data analyses and statistical tests were performed using SPSS for Windows (version 21) software. Associations between variables were determined using Spearman rank correlation and Mann-Whitney $U$ tests ( $p$-values of less than 0.05 were considered statistically significant). Data are given as means \pm standard deviations for continuous data or as percentages for discrete variables.

\section{RESULTS}

The prevalence of MEC was $92.3 \%$ (91.5\% in women and $93.3 \%$ in men). It was absent in 19 patients, unilateral in 44 and bilateral in 185 . It was present in $414(83.5 \%)$ of 496 temporal bones (84.7\% [n = $=210]$ on the right and $82.3 \%[n=204]$ on the left).

Patients were divided into three groups according to the diameter of the MEC (Table 1). The mean diameter was $1.58 \pm 0.86 \mathrm{~mm}$ (range: $0.1-4.8 \mathrm{~mm}$ ) on the right and $1.48 \pm 0.79 \mathrm{~mm}$ (range: $0.4-5 \mathrm{~mm}$ ) on the left side. The mean diameter of MEC was larger in men. A statistically significant correlation was detected between the diameter of the MEC and gender $(p<0.05)$. However, the diameter of the MEC was not correlated with age.
A total of 727 MFs were detected on 3D images. One hundred and forty seven (66 on the right and 81 on the left) of them did not connect with a canal on thin section axial MDCT images and were considered as pits. A total of 11 foramina were detected on axial MDCT images, but were not visible on 3D images. The largest MF was $6 \mathrm{~mm}$ (mean $1.92 \pm 1.02$ ) on the right and $6.3 \mathrm{~mm}$ (mean $1.84 \pm 0.98$ ) on the left. MFs were divided into four groups according to their diameters (Table 2). The number of the MFs changed from absent to triple (Fig. 2A-C). Single MF was seen in 263 (53\%) temporal bones. It was absent in $82(16.5 \%)$, double in $125(25.2 \%)$ and triple in 26 $(5.2 \%)$ temporal bones. The number of the MFs was demonstrated in Table 3.

\section{DISCUSSION}

The MEC transmits the MEV, which is one of the major posterior fossa emissary veins and allows anastomosis of the sigmoid sinus to the suboccipital venous plexus $[11,17,19,22]$. There may be multiple MEVs and various sizes of MEV have been described in previous studies [3, 11, 22]. Although it is generally small and has slow blood flow, it may be dilated in patients with high-flow vascular malformations or occluded internal jugular veins $[10,16,17,22]$. We evaluated the prevalence and morphometric features of MEC based on thin-section head and temporal bone MDCT images.

At least one MEC was present in 229 (92.3\%) of patients. It was bilateral in 185 (74.6\%) patients and 


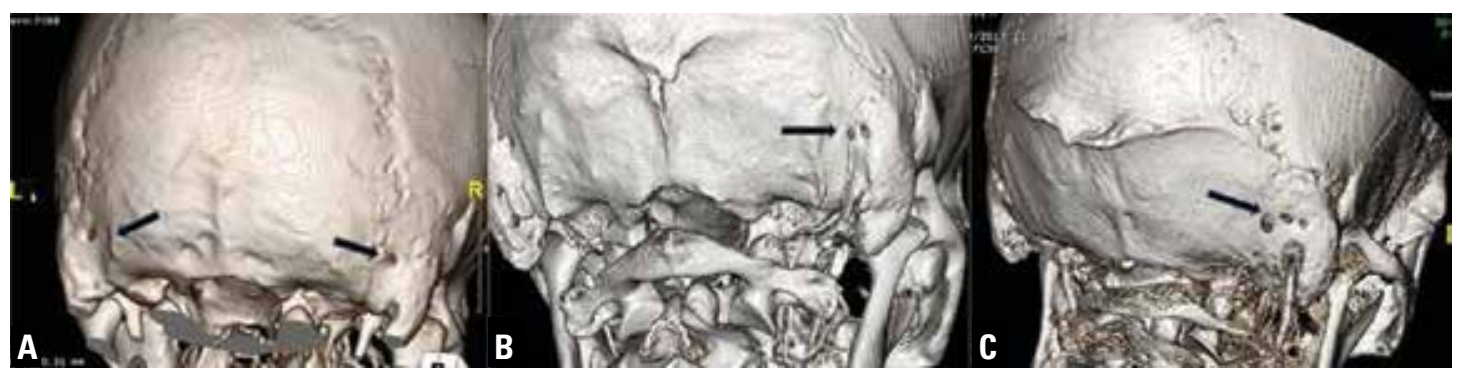

Figure 2. Three-dimensional volume rendered computed tomography image showing (A) single, $(\mathbf{B})$ double and $(\mathbf{C})$ triple mastoid foramen (arrows) on temporal bone.

Table 3. The number of mastoid foramina in both sides

\begin{tabular}{lccc}
\hline Morphology of mastoid foramina & \multicolumn{3}{c}{ Number of temporal bones (frequency; \%) } \\
\cline { 2 - 4 } & Right side $(\mathbf{n}=\mathbf{2 4 8})$ & Left side (n= 248) & Total (n= 496) \\
\hline Absent & $38(15.3 \%)$ & $44(17.7 \%)$ & $82(16.5)$ \\
Single & $132(53.2 \%)$ & $131(52.8 \%)$ & $263(53.0 \%)$ \\
Double & $64(25.8 \%)$ & $61(24.6 \%)$ & $125(25.2 \%)$ \\
Triple & $14(5.6 \%)$ & $12(4.8 \%)$ & $26(5.2 \%)$ \\
\hline
\end{tabular}

Table 4. Prevalence of mastoid emissary veins in different studies with the data from the literature

\begin{tabular}{lc}
\hline Reference & $\begin{array}{c}\text { Frequency in temporal bones } \\
(\%)\end{array}$ \\
\hline Koesling et al. [13] & $82^{\mathrm{B}}$ \\
Murlimanju et al. [18] & $89.6^{\mathrm{R}}-93.8^{\mathrm{L}}$ \\
Reis et al. [22] & $89^{\mathrm{B}}$ \\
Louis et al. [16] & $98^{\mathrm{R}}-72^{\mathrm{L}}$ \\
Kim et al. [11] & $81^{\mathrm{R}}-74^{\mathrm{L}}$ \\
Present study & $84.7^{\mathrm{R}}-82.3^{\mathrm{L}}$ \\
\hline
\end{tabular}

$\mathrm{R}$ — right side; $\mathrm{L}$ — left side; $\mathrm{B}$ - both sides

unilateral in 44 (17.7\%). Koesling et al. [13] were the first to use high-resolution axial CT to investigate mastoid emissary and other small canals of the temporal bone. They observed MEV in $82 \%$ of the temporal bones. This was similar to our study, in which MEC was present in $83.5 \%$ of temporal bones. The MEC was slightly more frequent on the right side, but the difference was not significant $(84.7 \%$ on the right and $82.3 \%$ on the left). Other investigators also reported a right side predominance, but with statistically significant difference $[11,16]$. In these previous studies, MEVs were evaluated on cadaveric specimens and no information regarding the prevalence according to gender was given. With our study, MEC was slightly more frequent in men $(91.5 \%$ of women and $93.3 \%$ of men). Pereira et al. [20] reported similar results. The comparison of the frequency of MEVs on temporal bones in the present study and the previous reports is represented in Table 4. Murlimanju et al. [18] reported that there was no significant difference among the races with respect to the prevalence of MEVs. In a recent study by Pekcevik et al. [21] the prevalence of MEV was $77.7 \%$ on CT angiograms. It was bilateral in $49.4 \%$ and unilateral in $28.3 \%$ and was more common on the left side. The relatively higher frequency reported by Koesling et al. [13] was explained by Koesling's results concerning the prevalence of the bony canal, not the emissary vein [13]. However, Pekcevik et al. [21] did not mention any bony canal not transmitting an emissary vein.

The presence and the diameter of MEV are important. Its existence and size should be studied on the thin-slice bone window CT scan before surgical procedures involving the posterior fossa or the mastoid region [11, 22]. Unexpected bleeding due to emissary vein injury is an important complication of mastoidectomy, epitympanectomy and suboccipital craniotomies. The amount of bleeding is related to the size of the emissary vein encountered and can be life-threatening. MEVs $3.5 \mathrm{~mm}$ in 
diameter or greater are considered large [1]. Other consequences of emissary vein bleeds are epidural and subdural hematoma formations [6]. Venous anomalies of the internal jugular vein and sigmoid sinus are not uncommon in patients with otitis and labyrinthine dysplasia and may lead to enlargement of the emissary veins [7]. These patients are candidates for surgery and the description of these veins should be systematically included in temporal bone $\mathrm{CT}$ reports to provide awareness of the surgeon to avoid injuring these veins. A similar consideration should be paid in patients who are candidates for cochlear implantation [24]. Furthermore, surgical materials such as bone wax applied to the bleeding foramina for achieving haemostasis, may predispose one to thrombosis of the sigmoid sinus and venous infarction [4, 8]. Another risk for venous ischaemic and haemorrhagic consequences is ligation of the emissary veins during skull base tumour surgery as the emissary veins may be the primary venous outflow pathway in patients with occluded internal jugular veins. This is also important in craniosynostosis surgery, since MEV may be the only drainage route of the brain in these patients [11]. Two cases of cerebellar infarction and one death resulting from coagulation of the MEV during skull base surgery have been reported [9]. Air embolism due to excessive traction of the emissary vein/veins is another significant risk in patients who are placed in the semi-sitting and lateral lying position on the operating table. Sufficient amounts of air to cause death can enter through an enlarged vein [15, 22]. Therefore, preoperative identification and reporting of these veins [12] are important to avoid undesirable morbidity and mortality. Sometimes a dilated MEV may clarify the patient's symptoms. A few case reports have shown that a dilated MEV may be the sole reason of pulsatile tinnitus [14].

Knowledge of anatomy of the cranial emissary veins provides some advantages during endovascular treatments and posterior fossa surgeries. The MEV is a reliable landmark to estimate the location of the sigmoid sinus and the cranial nerves during transcondylar and retrosigmoid approaches. Dural arteriovenous malformations in the transverse or sigmoid sinuses may be embolised through the MEV if the sinus is occluded in the proximal or distal side [23].

Previous studies based on cadaveric specimens evaluated the diameter of the outer foramen, the MF. The diameter of the MEC was evaluated with studies based on thin-section CT scans. We measured the diameter of the largest canal and the largest outer foramen on each temporal bone on axial $\mathrm{CT}$ images. We adopted the term "the diameter of MEC" to refer to the diameter of the largest MEC on each temporal bone. The diameter of MEC ranged from $0.1 \mathrm{~mm}$ to $4.8 \mathrm{~mm}$ (mean $1.58 \pm$ $\pm 0.86 \mathrm{~mm}$ ) on the right and from $0.4 \mathrm{~mm}$ to $5 \mathrm{~mm}$ (mean $1.48 \pm 0.79$ ) on the left. More than $77 \%$ of MECs were larger than $1 \mathrm{~mm}$, in contrast to Koesling et al. [13] who reported over $90 \%$ of the MECs to be less than $1 \mathrm{~mm}$ in diameter. The mean diameter of the MF was $1.92 \pm 1.02 \mathrm{~mm}$ on the right and $1.84 \pm 0.98 \mathrm{~mm}$ on the left and was larger than that of the canal itself. Our results were in accordance with more recent reports which have shown average diameters of $2-3.5 \mathrm{~mm}$ [11, 22]. Kim et al. [11] deemed the vessel to be large enough to warrant ligation instead of controlling via electrocautery, if the diameter of the MF was larger than $2.5 \mathrm{~mm}$. With our study, $53(10.7 \%)$ temporal bones had at least one MEC larger than $2.5 \mathrm{~mm}$. Louis et al. [16] reported a larger average diameter $(3.5 \mathrm{~mm})$ for MF. They found no significant difference between gender and age with regard to morphometry of MF. In our study, the diameter of MEC was not correlated with age, but was significantly larger in men. The largest MF in our study was $6.3 \mathrm{~mm}$. Only $2.4 \%$ of MFs were larger than $4 \mathrm{~mm}$ and $4.8 \%$ of MECs were larger than $3 \mathrm{~mm}$ (Tables 1, 2).

The MFs may be single or multiple. A total of $727 \mathrm{MFs}$ were detected on 3D images, of which 147 (66 on the right and 81 on the left) did not connect with a canal on thin section axial MDCT images and were defined as pits, not foramina. Similarly, Reis et al. [22] described a rudimentary MFs not pierced by a vessel in 2 of the 18 cadaveric hemicraniums. The number of MFs in our study varied from absent to triple (Table 3). Single MF was the most frequent type. It was absent in $16.5 \%$ of temporal bones and triple MF were observed in only $5.2 \%$ of temporal bones. Louis et al. [16] reported 3 or more MF in $29 \%$ of temporal bones on the right side and $19 \%$ of temporal bones on the left. They reported no significant correlation between the number of MFs and the diameter. We did not investigate the correlation of the number and the diameter of MFs.

\section{Limitations of the study}

The limitation of present study is that all examinations were performed without contrast injection and the MEV itself could not be seen. Based on previous studies assessing MEV on cadaveric specimens and 
dry skulls, we assumed that MF and MEC indicated the topography of MEV. Furthermore, in a recently published study, MEVs were assessed on cranial CT angiographies and the authors used "MEV canal" as a synonym of "MEV" [21].

\section{CONCLUSIONS}

In conclusion, anatomic knowledge and understanding of the physiology of craniocervical venous drainage is necessary to avoid surgical complications. The radiologist and surgeons not only should be familiar with emissary veins and their variations to differentiate pathological structures from normal, but also they should be aware of their clinical importance. MDCT is a quick and reliable method for evaluating mastoid emissary veins.

\section{Acknowledgements}

We wished to acknowledge M. Arslan, MD, from the Department of Public Health, Medical School, Balıkesir University, for his contribution in the statistical analysis.

\section{REFERENCES}

1. Calligas JP, Todd NW Jr (2014) Hemorrhage from large mastoid emissary vein: pedicled, rotated, indented, periosteal-galeal flap. Laryngoscope, 124: 551-553. doi: 10.1002/lary.24259.

2. Chauhan NS, Sharma YP, Bhagra T, Sud B (2011) Persistence of multiple emissary veins of posterior fossa with unusual origin of left petrosquamosal sinus from mastoid emissary. Surg Radiol Anat, 33: 827-831. doi: 10.1007/ s00276-011-0822-x.

3. Cheatle $A$ (1925) The mastoid emissary vein and its surgical importance. Proc R Soc Med, 18: 29-34.

4. Crocker M, Nesbitt A, Rich P, Bell B (2008) Symptomatic venous sinus thrombosis following bone wax application to emissary veins. Br J Neurosurg, 22: 798-800. doi: 10.1080/02688690802256399.

5. Freire AR, Rossi AC, De Oliveira VCS, Prado FB, Caria PHF, Botacin PR (2013) Emissary foramens of the human skull: anatomical characteristics and its relations with clinical neurosurgery. Int J Morphol, 31: 287-292.

6. Garza-Mercado R (1983) Extradural hematoma of the posterior cranial fossa. Report of seven cases with survival. J Neurosurg, 59: 664-672.

7. Giesemann AM, Goetz GF, Neuburger J, Lenarz T, Lanfermann $\mathrm{H}$ (2011) Persistent petrosquamosal sinus: high incidence in cases of complete aplasia of the semicircular canals. Radiology, 259: 825-833. doi: 10.1148/ radiol.11101466.

8. Hadeishi H, Yasui N, Suzuki A (1995) Mastoid canal and migrated bone wax in the sigmoid sinus: technical report. Neurosurgery, 36: 1220-1223.

9. Hoshi M, Yoshida K, Ogawa K, Kawase T (2000) Hypoglossal neurinoma: two case reports. Neurol Med Chir, 40: 489-493.
10. Keskil S, Gözil R, Calgüner E (2003) Common surgical pitfalls in the skull. Surg Neurol, 59: 228-231.

11. Kim LK, Ahn CS, Fernandes AE (2014) Mastoid emissary vein: anatomy and clinical relevance in plastic and reconstructive surgery. J Plast Reconstr Aesthet Surg, 67: 775-780. doi: 10.1016/j.bjps.2014.03.002.

12. Kiritsi O, Noussios G, Tsitas G, Chouridis P, Lappas D, Natsis K (2011) Anatomical variants of the emissary veins: unilateral aplasia of both the sigmoid sinus and the internal jugular vein and development of the petrosquamosal sinus. A rare case report. Folia Morphol, 70: 305-308.

13. Koesling S, Kunkel P, Schul T (2005) Vascular anomalies, sutures and small canals of the temporal bone on axial CT. Eur J Radiol, 54: 335-343.

14. Lee SH, Kim SS, Sung KY, Nam EC (2013) Pulsatile tinnitus caused by a dilated mastoid emissary vein. J Korean Med Sci, 28: 628-630. doi: 10.3346/jkms.2013.28.4.628.

15. Lipow HW, Rickham PP (1956) An extracranial varix of the sigmoid sinus. J Pediatr, 49: 465-469.

16. Louis RG Jr, Loukas M, Wartmann CT, Tubbs RS, Apaydin N, Gupta AA, Spentzouris G, Ysique JR (2009) Clinical anatomy of the mastoid and occipital emissary veins in a large series. Surg Radiol Anat, 31: 139-144. doi: 10.1007/ s00276-008-0423-5.

17. Mortazavi MM, Tubbs RS, Riech S, Verma K, Shoja MM, Zurada A, Benninger B, Loukas M, Cohen Gadol AA (2012) Anatomy and pathology of the cranial emissary veins: a review with surgical implications. Neurosurgery, 70: 1312-1318. doi: 10.1227/NEU.0b013e31824388f8.

18. Murlimanju BV, Chettiar GK, Prameela MD, Tonse M, Kumar N, Saralaya VV (2014) Mastoid emissary foramina: an anatomical morphological study with discussion on their evolutionary and clinical implications. Anat Cell Biol, 47: 202-206. doi: 10.5115/acb.2014.47.3.202.

19. Okudera T, Huang YP, Ohta T, Yokota A, Nakamura $Y$, Maehara F, Utsunomiya H, Uemura K, Fukasawa H (1994) Development of posterior fossa dural sinuses, emissary veins and jugular bulb: morphological and radiologic study. Am J Neuroradiol, 15: 1871-1883.

20. Pereira GA, Lopes PT, Santos AM, Pozzobon A (2013) Study of landmarks in dried skulls in a Brazil population. J Morphol Sci 30: 94-97.

21. Pekcevik Y, Sahin H, Pekcevik R (2014) Prevalence of clinically important posterior fossa emissary veins on $C T$ angiography. J Neurosci Rural Pract, 5: 135-138. doi: 10.4103/0976-3147.131654.

22. Reis CV, Deshmukh V, Zabramski JM, Crusius M, Desmukh P, Spetzler RF, Preul MC (2007) Anatomy of the mastoid emissary vein and venous system of the posterior neck region: neurosurgical implications. Neurosurgery, 61: 193-200. doi: $10.1227 / 01$.neu.0000280095.14361.82.

23. Rivet DJ, Goddard JK, Rich KM, Derdeyn CP (2006) Percutaneous transvenous embolization of a dural arteriovenous fistula through a mastoid emissary vein. Technical note. J Neurosurg, 105: 636-639.

24. Sunkaraneni VS, Banerjee A, Gray RF (2004) Subdural haematoma: a complication of cochlear implantation. J Laryngol Otol, 118: 980-982. 